\title{
Brûlures orales iatrogènes de nature chimique : Présentation de deux cas, revue de la littérature et proposition de conduite à tenir.
}

\section{Genest $\mathbf{S}^{1}$, Boussouak $\mathrm{A}^{1}$, Lan $\mathrm{R}^{1,2}$, Catherine $\mathrm{JH}^{1,2}$}

1. Service d'Odontologie, Département de Chirurgie Orale, Centre Hospitalier Universitaire de la Timone - Marseille 2. UMR 7268 ADES, Aix-Marseille/EFS/CNRS - Marseille

\section{Introduction}

Les brûlures iatrogènes de la cavité buccale d'origine chimique peuvent être à l'origine de lésion allant de la desquamation épithéliale (1) à la microstomie et ankylose linguale (2). Elles peuvent être causées par des produits utilisés en dentisterie, comme le paraformaldéhyde, l'hydroxyde de calcium ou encore l'hypochlorite de sodium. On retrouve notamment des lésions érosives, nécrotiques, ulcératives, associées à une sécheresse buccale et des douleurs avec dysphagie.

\section{Matériels et méthodes}

Nous présentons ici le cas d'une mère et de sa fille présentant des brûlures buccales et labiales survenues à la suite de soins dentaires. L'examen exo-buccal mettait en évidence la présence de croûtes sur les commissures labiales avec une limitation de l'ouverture buccale. L'examen endo-buccal objectivait la présence de colorations jaunâtres associées à des plages érythémateuses et des lésions érosives de 5 à $8 \mathrm{~mm}$ de diamètre avec induration à la palpation. Des plaques blanchâtres se détachant à la précelle et des lésions bulleuses en vestibulaire étaient aussi retrouvées. Le produit de désinfection (contenant du peroxyde d'hydrogène à $1.14 \%$ et du nitrate d'argent à $0.1 \%$ ) du circuit d'eau du fauteuil dentaire a été mis en cause. La prise en charge a été l'exérèse des lambeaux détachables pour examen anatomopathologique qui a permis de poser un diagnostic différentiel avec des maladies auto-immunes. Une revue de la littérature a été réalisée afin de rechercher des cas similaires et proposer une standardisation de prise en charge. Sur 92 articles trouvés, 13 ont été retenus selon la méthode PRISMA.

\section{Résultats}

L'historique détaillé des circonstances d'apparition des lésions muqueuses et une revue des antécédents médicaux des patientes ont permis de différencier l'origine chimique de l'origine auto-immune, bien qu'un caractère génétique aurait aussi pu être suspecté en raison du lien de parenté.

Les cas retrouvés dans la littérature étaient liés au mauvais usage d'un agent chimique ou à des cas de démence ou tentatives de suicides par ingestion de produits caustiques. Les durées de cicatrisation rapportées étaient rapides, en moyenne 11 jours (de 7 à 28 jours). Des limitations d'ouverture buccale par formation de brides cicatricielles, pouvant causer une dysphagie progressive, de microstomie avec ankylose linguale et un cas d'œdème épiglottique asphyxiant engageant le pronostic vital ont également été rapportés. (3)

\section{Discussion}

Les brûlures orales chimiques d'origines iatrogènes restent rares et sont peu décrites dans la littérature, ce qui peut déstabiliser un praticien face à l'hétérogénéité des lésions. II n'existe actuellement aucun protocole de prise en charge pour ce types de brûlures. Etre vigilant face aux produits utilisés, rassurer le patient et suivre la cicatrisation restent la meilleure conduite à tenir. Néanmoins, d'un point de vue médico-légal, la rédaction d'un certificat médical initial est fortement conseillée. En fonction de l'importance des lésions et de la gêne occasionnée, le patient peut demander un pretium doloris en guise de compensation.

lanromain@live.fr

\section{Références}

(1) Deo SP, Shetty P. Accidental chemical burns of oral mucosa by herbicide. JNMA J Nepal Med Assoc, 2012; 52(185): 40-42.

(2) Su YT, Yeh CJ, et Ou CT. The management of chemically burned oral tissue - A case report. Zhonghua Ya Yi Xue Hui Za Zhi Chinese Dental Journal, 1988; 7(3): 128-133

(3) Ribeiro ACP et al. Formalin Burn. British Dental Journal, 2010; 209(1): 4.

(C) The authors, published by EDP Sciences. This is an Open Access article distributed under the terms of the Creative Commons Attribution License 4.0 (http://creativecommons.org/licenses/by/4.0/) 